\title{
notes
}

\section{Dosimétrie des personnes exposées aux rayonnements dans l'exercice de leur profession en Suisse}

\author{
Méthodes et résultats \\ $10^{\mathrm{e}}$ rapport de la Commission fédérale de la protection contre \\ les radiations pour l'année 1985, établi par le groupe d'experts \\ pour la dosimétrie*
}

(Manuscrit reçu le 23 avril 1987)

\section{RÉSUME}

Cette note présente le bilan dosimétrique des irradiations professionnelles en Suisse pour l'année 1985. Ce document est publiè chaque année depuis 1975 par l'Office fédéral de la santé publique (Beine).

\section{ABSTRACT}

The dose records for occupational exposures in Switzerland are presented for the year 1985 . The report has been published by the Office fédéral de la santé publique (Federal office for public health) in Bern every year since 1975.

\section{INTRODUCTION}

La Commission fédérale de la protection contre les radiations a constitué en 1970 un groupe d'experts pour la dosimétrie individuelle, comprenant des spécialistes appartenant aux instances de contrôle (OFSP, DSN, CNA), à des institutions publiques et à des entreprises privées.

Le rapport de la $10^{\mathrm{e}}$ année de fonctionnement du groupe est l'occasion de présenter les travaux effectués et de faire une analyse rétrospective des résultats obtenus.

- S. Prêtre, président, Division principale de la sécurité des installations nucléaires DSN (HSK), CH 5303 Würenlingen; A. Auf der Maur, A. Donath, J. Dutrannois, H. Lengweiler, H. Lüthy. B. Michaud, Th. Samuel, J.F. Valley, Ch. Wernly, membres. 
La mise en vigueur en 1981, par le Conseil fédéral, de l'ordonnance sur l'homologation et l'exploitation des services de dosimétrie individuelle (ordonnance sur la dosimétrie) représente un résultat important des travaux préparatoires effectués durant plusieurs années par le groupe.

La participation aux intercomparaisons organisées annuellement, et jusqu'alors à titre facultatif, par le groupe d'experts devient une obligation pour les services de dosimétrie, Ceux-ci doivent être à même de mesurer les doses d'irradiation situées entre 50 mrem ( $500 \mu \mathrm{Sv}$ ) et 500 rem (5 Sv) avec une précision située entre plus $50 \%$ et moins $30 \%$ en routine.

Parallèlement aux développements dans le domaine de la réglementation, la technique de la dosimétrie individuelle a progressé au cours de ces dernières années. Alors que la dosimétrie par film était auparavant le système le plus répandu, pour la première fois en 1982 plus de la moitié des personnes professionnellement exposées aux radiations étaient contrôlées à l'aide de dosimètres thermoluminescents (TLD); aujourd'hui la répartition est la suivante: $8 \bar{T} \%$ TLD, $10 \%$ dosimètres à film et $3 \%$ dosimètres au verre phosphaté.

\section{SERVICES DE DOSIMÉTRIE INDIVIDUELLE}

La mesure des doses individuelles a été effectuée par les services homologués suivants: Organisation européenne pour la recherche nucléaire (CERN), Genève; Comet Technik SA (COMET), Liebefeld; Institut fédéral de recherche en matière de réacteurs (EIR), Würenlingen; Institut de radiophysique appliquée (IRA), Lausanne; Centrale nucléaire de Beznau (KKB), Döttingen; Centrale nucléaire de Gösgen (KKG), Däniken; Centrale nucléaire de Leibstadt (KKL), Leibstadt; Centrale nucléaire de Mühleberg (KKM), Mühleberg; Prof. Dr. H. Lüthy (LY), Riehen; Service de dosimétrie individuelle (PEDOS), Bremgarten-Berne; Service cantonal de contrôle des irradiations ( $\mathrm{SCCl}$ ), Genève; Caisse nationale d'assurance en cas d'accidents (SUVA), Lucerne.

Les méthodes de mesure ainsi que le nombre de personnes professionnellement exposées aux radiations, surveillées par les différents services, sont donnés au tableau I.

\section{INTERCOMPARAISONS}

Durant l'exercice 1985, l'organisation de la $11^{\mathrm{e}}$ intercomparaison dosimétrique concernant l'irradiation externe a été confiée au CERN.

Les dosimètres ont été irradiés dans les conditions les plus réalistes possibles, à savoir: rayonnement provenant de diverses directions dans un champ anisotrope, large spectre énergétique, dosimètre placé sur un fantôme, doses situées entre 100 et 500 mrem (entre 1 et $5 \mathrm{mSv}$ ). L'irradiation habituelle de référence à l'aide du rayonnement du césium 137 a également èté effectuée.

Tous les résultats ont été situés à l'intérieur des limites de $-30 \%$ à $+50 \%$ fixées dans l'ordonnance sur la dosimétrie. 


\section{IRRADIATION EXTERNE}

\subsection{Dose au corps entier par irradiation externe}

L'irradiation externe est mesurée à l'aide de dosimètres portés par les personnes professionnellement exposées aux radiations. La contribution de I'irradiation naturelle est déduite systématiquement. Les doses au corps entier, ventilées suivant le domaine d'activité, sont données au tableau II, avec indication du nombre de personnes et de la dose annuelle.

Pour la première fois en 1985 , le nombre de personnes soumises au contrôle dosimétrique a dépassé 50 000. Parmi les 50980 personnes surveillées, deux tiers travaillent dans le domaine médical. Un seul dépassement de la limite annuelle de dose pour l'irradiation externe a été constaté (cf. paragraphe 6).

Les doses collectives, ventilées suivant le domaine d'activité et l'intervalle de dose, sont indiquées au tableau III. La dose collective, c'est-à-dire la somme des doses individuelles de toutes les personnes professionnellement exposées aux radiations, s'élève pour 1985 en Suisse à 1838 homme-rem $(18,38$ homme-Sv). Les contributions des différents secteurs sont les suivantes: centrales nucléaires $66 \%$, recherche $17 \%$, médecine $13 \%$ et services publics $4 \%$.

\subsection{Doses partielles par irradiation externe}

Les doses aux mains sont mesurées par thermoluminescence dans 6 services de dosimétrie individuelle. Le tableau IV donne par secteur et par intervalle de dose la répartition des valeurs enregistrées. La dose aux mains pour les personnes professionnellement exposées aux radiations est limitée à 75 rem par année (750 mSv). Parmi les 795 personnes surveillées, seules 2 personnes ont dépassé le tiers de cette limite. La valeur maximale mesurée est de 60 rem (600 mSv).

Les doses à la peau sont déterminées à l'aide de dosimètres conçus spécialement. Quelques services disposent de tels dosimètres. La limite annuelle est de $30 \mathrm{rem}(300 \mathrm{mSv})$. La contribution des rayonnements de basse énergie étant faible, les doses à la peau ne dépassent guère les doses au corps entier.

\section{INCORPORATION}

Le calcul de la dose due à l'incorporation s'effectue en déterminant l'activité présente dans certains organes ou à l'aide de l'analyse des excrétions. Le résultat est exprimé sous forme de pourcentage de la limite annuelle d'incorporation du nucléide correspondant.

Les résultats de ces mesures sont donnés dans le tableau $\mathrm{V}$ pour les divers secteurs d'activité en fonction des pourcentages de la limite annuelle 
d'incorporation. En cas d'incorporation supérieure à $2 \%$ de cette limite, les nucléides incorporés sont mentionnés. En 1985, 3797 personnes au total ont été contrôlées. Deux dépassements ont été constatés (cf. paragraphe $6)$.

\section{DÉPASSEMENTS DES LIMITES ANNUELLES DE DOSE}

Trois dépassements des limites annuelles de dose ont eu lieu en 1985:

1. Un contrôleur de matériau exécutait des examens radiologiques avec une source de $50 \mathrm{Ci}(1,85 \mathrm{TBq})$ d'iridium 192 dans une zone barrée à l'intérieur de l'entreprise. La source d'irradiation se détacha du câble de commande à distance et demeura en position d'irradiation, alors que le contrôleur la supposait dans le récipient de protection et effectuait plusieurs prises de clichés. Après $45 \mathrm{~min}$ il se rendit compte de la situation et demanda l'aide d'un spécialiste pour remédier à la panne. Le dosimètre du contrôleur a indiqué une dose de $17 \mathrm{rem}(170 \mathrm{mSv})$. L'examen des aberrations chromosomiques a donné un résultat inférieur à la limite de détection de cette méthode qui est de $10 \mathrm{rem}(100 \mathrm{mSv})$. La dose de $17 \mathrm{rem}(170 \mathrm{mSv})$ a été portée sur le carnet de contrôle de la personne.

La CNA a étudié l'incident et a découvert que l'accouplement entre le porte-source et la corde permettant de sortir la source était usé et ne correspondait plus aux tolérances. Actuellement, la CNA contrôle tous ces dispositifs. Le dépassement de dose aurait pu être facilement évité si le contrôle de la position de la source avait été effectué, selon les prescriptions, lors de chaque retour dans le récipient de protection.

2. Un accident d'incorporation a eu lieu sur une installation industrielle pour le remplissage de tritium gazeux. Une erreur de manipulation, ainsi qu'une vitre de protection laissée ouverte par la personne concernée, ont conduit à une incorporation d'environ $1 \mathrm{Ci}(37 \mathrm{GBq})$ de $\mathrm{HTO}$ (limite annuelle:

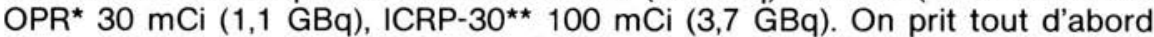
des mesures en vue d'accélérer l'élimination du tritium. A cet effet la personne séjourna 4 jours à l'hôpital. A partir des mesures d'urine effectuées durant les 50 premiers jours, l'équivalent de dose efficace calculé selon I'ICRP-30 conduit à une dose de 46 rem ( $460 \mathrm{mSv}$ ). Plusieurs mesures des aberrations chromosomiques ont donné une dose d'environ 38 rem $(380 \mathrm{mSv})$. Des examens médicaux complémentaires ont été négatifs. Ce cas, qui fait l'objet d'une évaluation scientifique, confirme que le calcul de dose, selon l'appendice 7 aujourd'hui dépassé de l'ordonnance sur la radioprotection, donnerait, avec 120 rem, une valeur exagérée. Entre-temps, l'installation a été équipée d'une protection technique contre les erreurs de manipulation de ce type.

3. Une entreprise envoya un collaborateur dans une filiale étrangère pour un stage dans le domaine du marquage de produits à l'iode 125 . Une incorporation, dont le déroulement ne peut être reconstruit exactement, eut lieu. La personne se blessa à un doigt lors du nettoyage d'une chapelle

* OPR: Ordonnance fédérale sur la protection contre les radiations.

**ICRP: Commission internationale de protection radiologique. 
isotopique; elle retira son gant et suça la plaie. On suppose qu'à cette occasion elle a incorporé une contamination des mains. L'incorporation ne fut découverte que le lendemain à l'aide du moniteur pour les mains et les pieds du laboratoire. A son retour en Suisse, l'activité en iode 125 dans la glande thyroïde fut mesurée régulièrement sur un moniteur étalonné. L'activité initiale de $22 \mu \mathrm{Ci}(814 \mathrm{kBq})$ dans la glande thyroïde diminua avec une période de 36 jours. La dose engagée dans la thyroïde est de 80 rem ( 800 $\mathrm{mSv}$ ), ce qui correspond à environ trois fois la valeur de la limite annuelle de I'OPR. Le seuil de la dose par organe selon l'ICRP-30, de 50 rem (500 mSv), est aussi dépassé. L'équivalent de dose efficace, paramètre significatif pour le risque stochastique selon l'ICRP 26, n'est cependant que de 2,4 rem (24 mSv).

\section{RÉTROSPECTIVE ET CONCLUSIONS}

Quelques évolutions et tendances de la dosimétrie individuelle en Suisse seront analysées dans le cadre d'une rétrospective des 10 dernières années.

Le nombre de personnes professionnellement exposées aux radiations a continuellement augmenté au cours des 10 dernières années. Ceci correspond d'une part à un accroissement réel de ce collectif, mais d'autre part c'est le fait d'une meilleure surveillance des personnes professionnellement exposées aux radiations, en particulier dans le domaine médical.

La dose collective, c'est-à-dire la somme des doses individuelles au corps entier de toutes les personnes professionnellement exposées aux radiations, est le meilleur paramètre décrivant la situation globale. Malgré l'augmentation du nombre des personnes surveillées au cours de ces 10 dernières années, on peut remarquer une tendance à la baisse de la dose collective, tendance qui peut être interprétée comme un succès de la radioprotection.

La diminution de la dose collective globale des personnes professionnellement exposées aux radiations est à attribuer aux domaines: "médecine", "recherche" et "industrie/services publics".

L'augmentation de la contribution des centrales nucléaires est due à l'augmentation des travaux de révision nécessaires pour les anciennes installations et à la mise en service de deux nouvelles centrales. En 1983, des travaux particuliers de révision ont dû être effectués à la centrale nucléaire de Beznau, travaux entraînant une augmentation de la dose collective.

Le nombre de personnes qui ont reçu une dose annuelle inférieure à 200 mrem ( $2 \mathrm{mSv}$ ) a augmenté de manière continue, comme le nombre total des personnes surveillées, alors que le nombre de personnes ayant reçu une dose supérieure à 200 mrem est en baisse.

Le groupe de personnes ayant reçu une dose supérieure à 200 mrem (2 mSv) par an (en 1985 ce groupe représentait 3,5\% des personnes surveillées) a accumulé, au cours de chacune des 10 années, environ $80 \%$ de la dose collective reçue par les personnes professionnellement exposées aux radiations. Ainsi, la contribution principale à la dose collective n'est pas constituée par sommation d'un très grand nombre de petites valeurs. 
Les doses liées à l'incorporation concernent peu de personnes. Dans la plupart des cas, il s'agit d'incorporation de tritium, radioélément utilisé pour la fabrication de peintures et de matériaux luminescents.

Tous les dépassements des limites annuelles font l'objet d'un examen particulier. Les 41 dépassements annoncés au cours de ces 10 dernières années (ce chiffre comprend également 4 cas d'incorporation) peuvent être répartis comme suit:

\begin{tabular}{|c|c|c|c|}
\hline & "faux" & "incertain" & "vrai" \\
\cline { 2 - 4 } 1976 & 3 & 7 & 0 \\
1977 & 0 & 2 & 3 \\
1978 & 0 & 6 & 2 \\
1979 & 1 & 4 & 1 \\
1980 & 0 & 0 & 1 \\
1981 & 0 & 0 & 1 \\
1982 & 0 & 1 & 0 \\
1983 & 0 & 3 & 2 \\
1984 & 1 & 0 & 0 \\
1985 & 0 & 0 & 3 \\
\hline
\end{tabular}

Le terme "faux" signifie que la personne n'a pas été irradiée. Dans les cas incertains, un dépassement ne peut être exclu. Sur l'ensemble des cas, un seul dommage a été constaté (brûlure à une main).

\section{CONCLUSIONS}

Sur la base des résultats de la dosimétrie individuelle, l'évolution de la radioprotection en Suisse au cours de ces 10 dernières années peut être considérée comme réjouissante.

Bien que le nombre des personnes professionnellement exposées aux radiations ait augmenté de manière continue, la dose collective reçue par ce groupe indique une tendance à la baisse. Ceci est particulièrement significatif, car le nombre d'installations produisant des rayonnements ionisants a sensiblement augmenté aux cours de ces 10 années.

Ce succès n'aurait certainement pas été obtenu, si l'on n'avait pas attaché l'importance qu'elle mérite à la dosimétrie des personnes professionnellement exposées aux radiations. A ce jour les instances de contrôle tiennent la surveillance physique bien en mains.

Nous remercions sincèrement ici $M$. H. FELBER (OFSP), qui a rédigé les procès-verbaux des séances et accompli de nombreux travaux d'organisation. M. H.J. PFEIFFER (DSN) a assisté le président du groupe d'experts dans différentes täches, tout en remplissant des fonctions de secrétaire scientifique. Le présent rapport a également bénéficié de la collaboration active de M. W. ZELLER (OFSP). Qu'ils trouvent ici nos remerciements cordiaux. 
NOTES

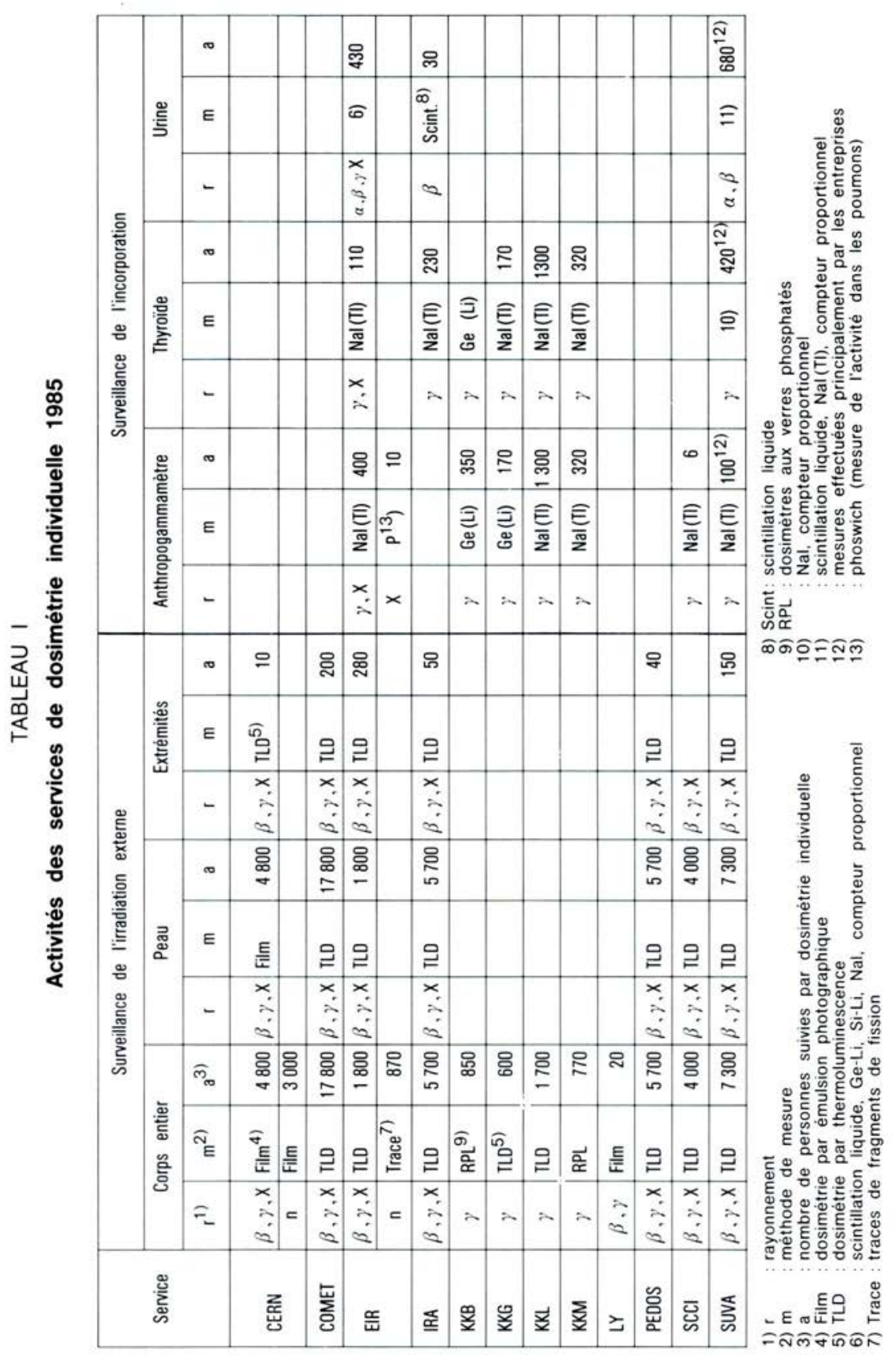

VOL. $22 \cdot \mathrm{N}^{\circ} 3$ 
NOTES

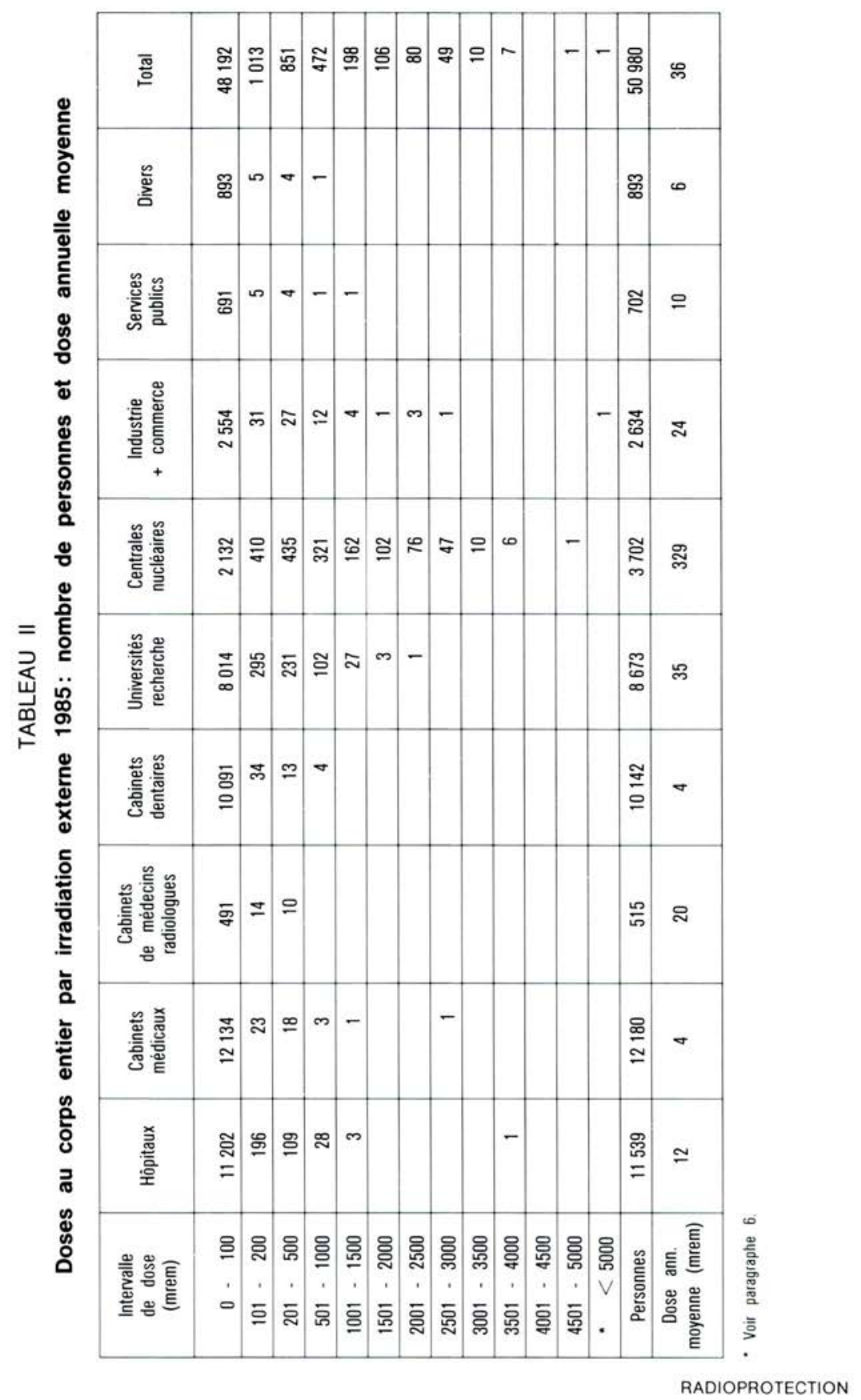


NOTES

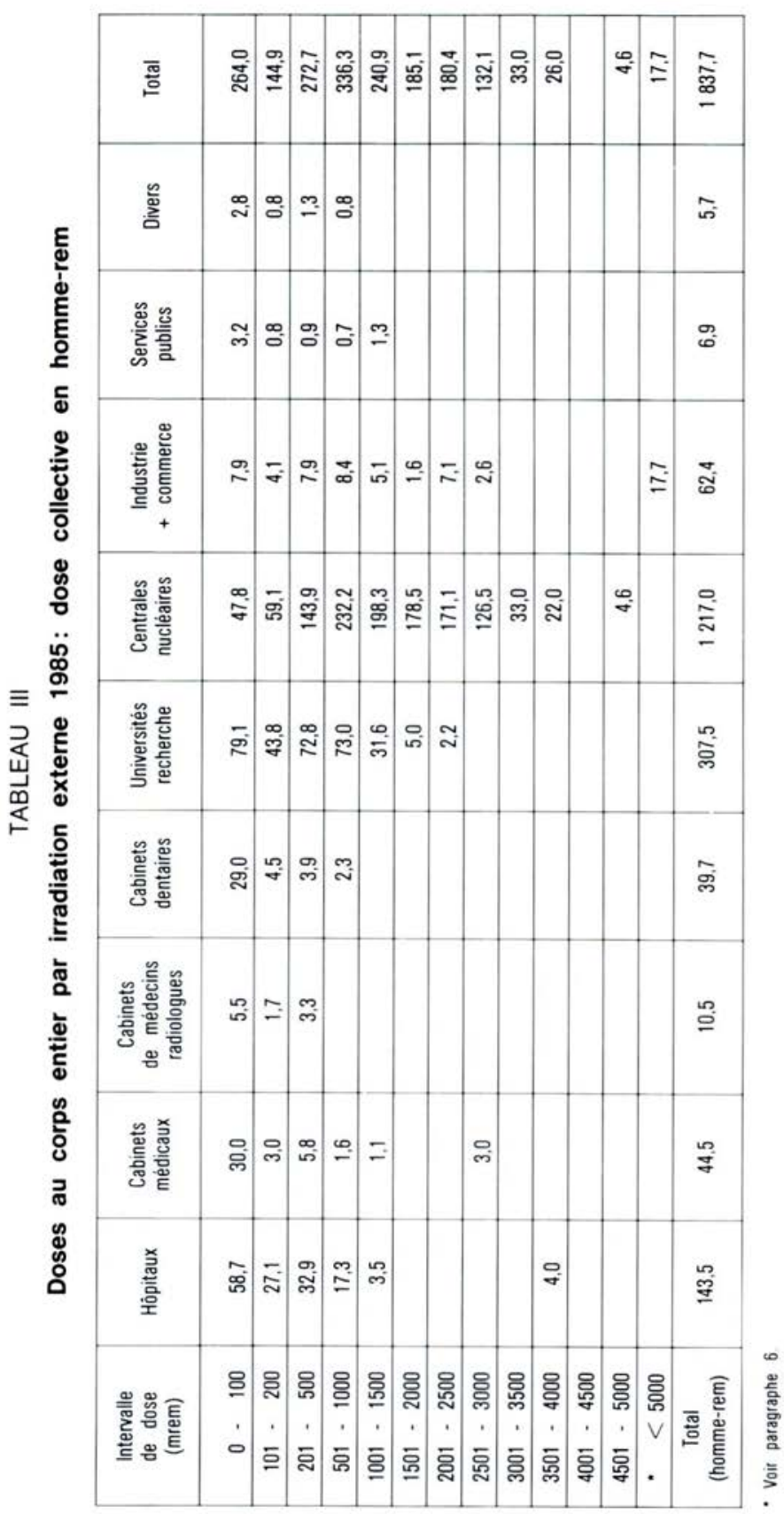

VOL. $22 \cdot \mathrm{N}^{\circ} 3$ 
NOTES

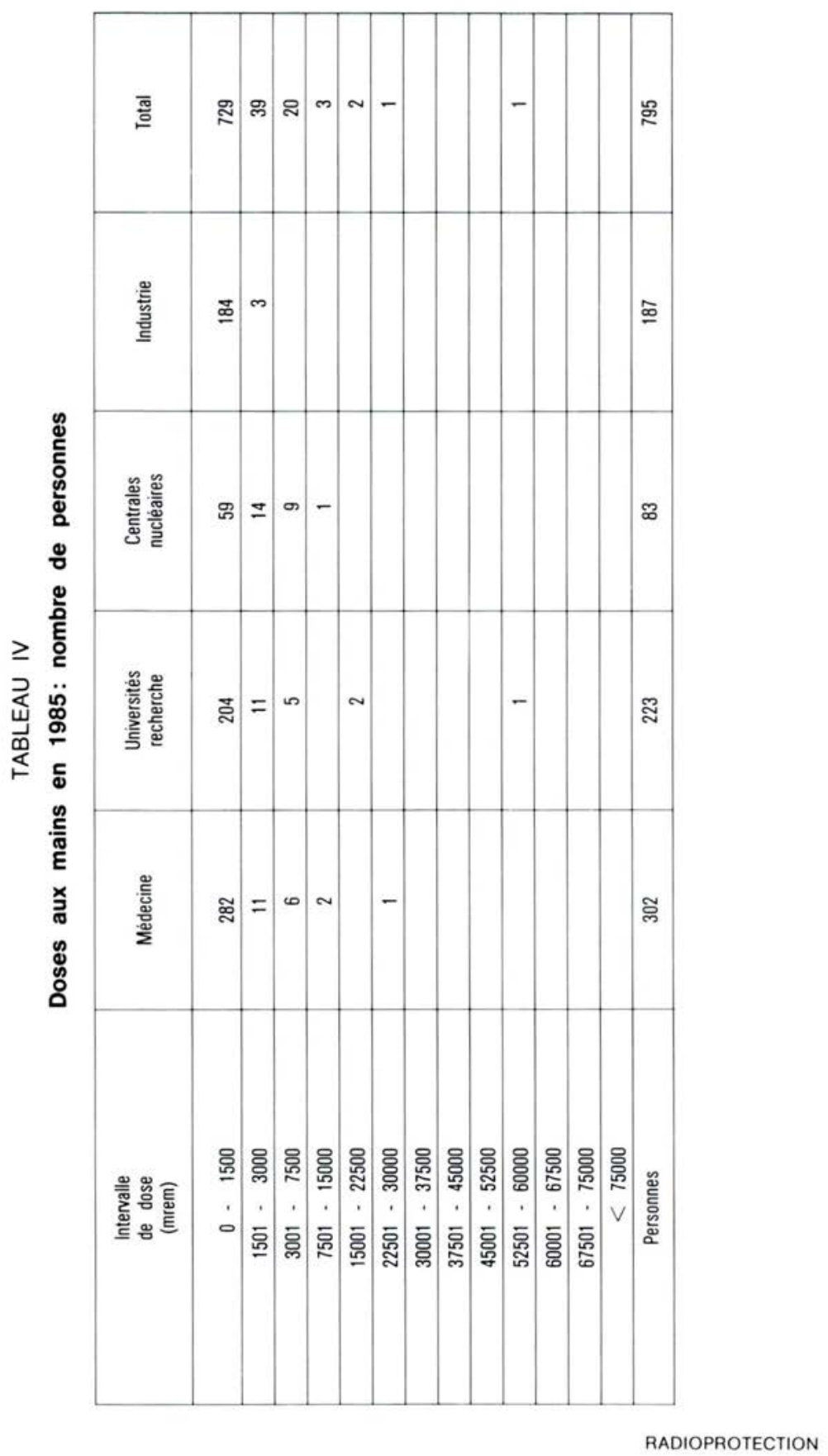


NOTES

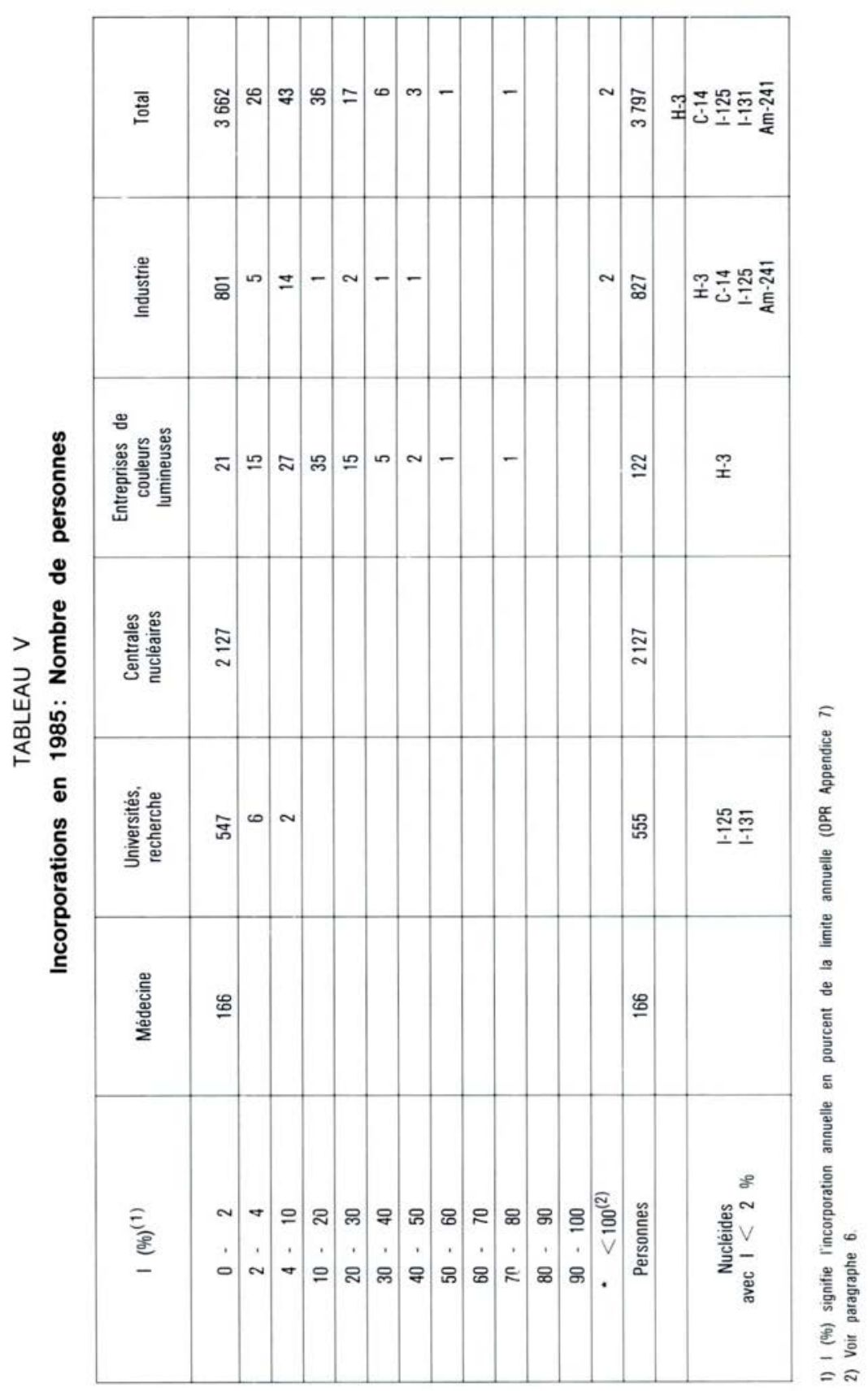

VOL. $22-\mathrm{N}^{\circ} 3$ 